\section{Outcome after systemic thrombolysis is predicted by age and stroke severity: an open label experience with recombinant tissue plasminogen activator and tirofiban}

\author{
Rüdiger J. Seitz,1,2,3 Judith Sukiennik,1 \\ Mario Siebler 1,4 \\ 1Department of Neurology, University \\ Hospital Düsseldorf, 2Biomedical \\ Research Centre, Heinrich-Heine- \\ University Düsseldorf, Germany; \\ 3Florey Neuroscience Institutes, \\ Melbourne, Australia; ${ }^{4}$ Department \\ of Neurology, Mediclin Fachklinik Rhein \\ Ruhr, Essen, Germany
}

\section{Abstract}

Stroke patients can recover upon intravenous thrombolysis but remain impaired in lacking recanalization. We sought to investigate the clinical effect of systemic thrombolysis with an intravenous bolus of $20 \mathrm{mg}$ recombinant tissue plasminogen activator (rtPA) and an infusion of body-weight adjusted tirofiban for 48 hours in acute stroke. This prospective, open label study, included 192 patients $(68 \pm 13$ years, $50 \%$ males) treated between 1 January 2005 and 31 December 2007. The neurological deficit was assessed with the National Institutes of Health stroke scale (NIHSS). Follow-up was performed using a telephone interview of modified Rankin Scale (mRS) and Barthel index. The site of cerebral artery occlusion was determined by computed tomography or magnetic resonance angiography. Data were analyzed by descriptive statistics and multiple regression analyses. Eighty-one percent of the patients had an infarct in the middle cerebral artery (MCA) territory and were severely affected with a median NIHSS of 10 . During treatment on the Stroke Unit the patients improved $(\mathrm{P}<0.0001)$ except for patients who deceased due to malignant infarction $(n=10)$ or cerebral haemorrhage $(n=6) ; 18$ percent deceased within 100 days which was predicted by older age $(76+10$ years, $\mathrm{P}<0.05)$ and more severe affection on admission $(\mathrm{P}<0.0001)$. Also, these patients more frequently had atrial fibrillation $(\mathrm{P}<0.03)$ than the surviving patients. The surviving patients had more frequently distal MCA occlusions and improved further $(\mathrm{P}<0.0001)$. At follow-up $48 \%$ of the patients had a mRS of 0 and 1. Similarly to intravenous thrombolysis with body-weight adjusted rtPA, poor prognosis was predicted by higher age, more severe neu- rological deficit at stroke admission, and a proximal MCA occlusion. Half of the surviving patients improved to no or minimal impairment.

\section{Introduction}

Systemic thrombolysis with the recombinant tissue plasminogen activator (rtPA) is the only approved treatment of acute ischemic stroke. It aims at rapid recanalization of the occluded cerebral artery affording neurological recovery. ${ }^{1}$ In recent years it was shown that early recanalization results in better neurological outcome and less lesion growth than lacking recanalization. ${ }^{2-4}$ However, the severity of the ischemic attack prior to thrombolytic treatment and a poor status of arterial collaterals have been described to predict a poor recovery. 5,6 On average, thrombolysis has been found to enhance the proportion of patients with a favorable outcome after ischemic stroke and to downsize the proportion of patients with severe neurological deficits. ${ }^{7-9}$ In fact, a large recent meta-analysis of different thrombolysis studies revealed that the application of alteplase rendered some 42 percent of patients with an excellent outcome [modified Rankin Scale (mRS) of 0 and 1 ] on day 90 as compared with 30 percent of patients receiving placebo. 10

Prompted by the results of platelet receptor inhibition in the management of patients with acute myocardial ischemia we have introduced a regime for systemic thrombolysis composed of an intravenous bolus of low dose rtPA in combination with a subsequent infusion of the nonpeptide GPIIb/IIIa platelet receptor antagonist tirofiban. ${ }^{11,12}$ The rational for a low dose of rtPA was to avoid intra- and extracranial hemorrhage, since symptomatic and fatal hemorrhages are a severe complication of a high dose of rtPA. ${ }^{13}$ Further, GPIIb/IIIa receptor antagonists selectively inhibit the platelet integrin IIb III fibrinogen receptor and thereby inhibit platelet aggregation. ${ }^{14,15}$ Since rtPA is known to induce a hypercoagulation following thrombolysis, ${ }^{16}$ tirofiban was expected to antagonize the hypercoagulation following rtPA administration and thereby help to maintain the cerebral blood vessels patent after thrombolysis. Likewise, it might help to disaggregate secondary thrombi resulting from the large thrombus in the initially occluded cerebral artery. We have shown previously that the combined use of low dose rtPA and tirofiban is safe and effective.12,17 Thus, we used this treatment as first line regime.

In this prospective mono-center, non-randomized study we analyzed the clinical data of the consecutive patients treated with a low bolus of rtPA and a subsequent infusion of tirofiban in our hospital in the years 2005
Correspondence: Rüdiger J. Seitz, Department of Neurology, University Hospital Düsseldorf Moorenstrasse 5, 40225 Düsseldorf, Germany. Tel. +49.211.8118974 - Fax: +49.211 .8118485 . E-mail: seitz@neurologie.uni-duesseldorf.de

Key words: brain infarct, stroke, thrombolysis, tirofiban, impairment.

Funding: the study was supported by the Competence Net Stroke of the BMBF.

Conflict of interests: the authors report no potential conflict of interests.

Received for publication: 28 September 2011.

Revision received: 11 June 2012.

Accepted for publication: 30 July 2012 .

This work is licensed under a Creative Commons Attribution NonCommercial 3.0 License (CC BYNC 3.0).

@CCopyright R.J. Seitz et al., 2012

Licensee PAGEPress, Italy

Neurology International 2012; 4:e9

doi:10.4081/ni.2012.e9

through 2007. We investigated four questions: i) what is the proportion of patients who benefit from this treatment? ii) what is the proportion of patients who do not benefit from this treatment? iii) what is the proportion of patients who expire after this stroke treatment? iv) what are predictors for poor or good recovery?

\section{Materials and Methods}

\section{Subjects}

All patients who were treated with systemic thrombolysis on the Stroke Unit of our institution between 1 January 2005 and 31 December 2007 were included. Criteria for inclusion into this study were:

an acute ischemic brain infarct,

documentation of a causal cerebral artery occlusion before thrombolysis using computed tomographic angiography (CTA) or magnetic resonance angiography (MRA), systemic thrombolysis with rtPA and tirofiban,

clinical evaluation on admission and at discharge from the Stroke Unit using the stroke scale of the National Institutes of Health (NIHSS), the mRS and Barthel index (BI), $18-21$

follow-up telephone questionnaire of the BI and $\mathrm{mRS}$, which was performed after at least 130 days (median 487 days, range 131-1056 days).

Patients were subjected either to computed 
tomography including CTA or multiparametric magnetic resonance imaging including a MRA depending on availability prior to thrombolysis.

Thrombolysis was performed in each patient with an intravenous bolus of $20 \mathrm{mg}$ rtPA within $3 \mathrm{~h}$ after stroke onset followed immediately by an intravenous infusion of tirofiban.12,17 Tirofiban was given in a body-weight adjusted dosage starting with a bolus of $0.4 \mu \mathrm{g} / \mathrm{kg}$ body weight/min for $30 \mathrm{~min}$ followed by continuous infusion of $0.1 \mu \mathrm{g} / \mathrm{kg}$ body weight/min for $48 \mathrm{~h}$. In addition, patients were treated according to their individual requirements. The treatment procedure and the study were approved by the Ethics Committee of the Heinrich-HeineUniversity Düsseldorf, Germany. The patients gave informed consent.

\section{Statistical analysis}

Statistical analysis was done using SPSS 10.1 for Windows (release 2005). Group comparisons were done using the two-tailed t-test. Evaluation of the neurological deficit and the impairment was assessed with the non-parametric MannWhitney test. Group comparison of the abnormalities in the cerebral arterial circulation was performed with the distribution free Wilcoxon's rank test. Multiple regression analyses were performed to single out the variables that accounted for the effect of thrombolysis.

\section{Results}

Of 2241 patients treated on the Stroke Unit of this institution in 2005 through 2007, 252 patients (11\%) were treated with systemic thrombolysis using intravenous rtPA and tirofiban. Fifteen patients were subjected to body weight adjusted rtPA or received a catheter intervention due to a stroke in the vertebrobasilar territory. The 192 patients $(70 \pm 13$ years, 50 percent males) matching the inclusion criteria of this study were severely impaired at stroke onset having a median NIHSS of 10 (range 2-27). 81\% of the patients had a middle cerebral artery (MCA) territory infarct. There were only few patients with a tandem occlusion of the internal carotid artery (ICA) and the MCA. On average the patients improved by 7 points on the NIHSS $(\mathrm{P}<0.0001)$ until discharge from the Stroke Unit (Table 1). The profound improvement was also apparent in the mRS, and BI (Table 1). The majority of the patients with a tandem occlusion of the ICA and MCA deceased. In contrast, the more peripheral the MCA occlusions occurred, the more patients survived (Figure 1). There was no difference among the subgroups concerning age, gender or vascular risk factors, apart from atrial fibrillation being more frequent in tandem occlusions of the ICA and MCA or at the bifurcation as compared to a MCA-branch $(\mathrm{P}<$
0.001). Twenty-three patients died with 6 patients due to an intracranial haemorrhage (3.1\%) and 10 patients suffering malignant brain infarction. After discharge eleven further patients deceased such that 34 patients (18\%) deceased within 100 days after stroke (Figure $2)$. Death was predicted by older age $(76 \pm 10$ years, $\mathrm{P}<0.05)$ and a more severe affection (mRS 5, P<0.0001). Also, the patients more frequently had atrial fibrillation $(\mathrm{P}<0.03)$ than the surviving patients. But none of the other vascular risk factors was predicting or different between the deceased or surviving patients (Table 1). The surviving patients improved further $(\mathrm{P}<0.0001)$ leaving $48 \%$ of study cohort with a mRS of 0 and 1 at follow-up, while $18 \%$ of the patients were still severely affected having a mRS of 4 or 5 (Figure 3 ).

\section{Discussion}

This study revealed that the combined use of an intravenous bolus of low dose rtPA and a subsequent body-weight adjusted infusion of tirofiban had an optimal outcome (mRS of 0 and 1) at follow-up in about 48 percent of our patients. In comparison to the large clinical trials of systemic application of rtPA this was clearly better than in the placebo group and similar to the thrombolysis group. ${ }^{10}$ Note, that the patients in this study had a median NIHSS at treatment onset of 10 being as severely affected as the patients in the ECASS 3 study. ${ }^{1}$ Importantly, the patients who benefitted from our treatment regime were younger and less severely affected at stroke onset than those

Table 1. Characteristics of the 192 patients.

\begin{tabular}{lc}
\hline Age (years \pm SD) & $70 \pm 13$ \\
Male/Female (n) & $95 / 97$ \\
\hline NIHSS, admission (median, range) & $10(2-27)$ \\
BI, admission (median, range) & $30(0-100)$ \\
\hline mRS, admission (median, range) & $4(0-5)$ \\
NIHSS, discharge (median, range) & $3(0-23)^{*}$ \\
\hline BI, at discharge (median, range) & $80(0-100)^{*}$ \\
mRS, at discharge (median, range) & $3(0-6)^{*}$ \\
\hline Arterial hypertension (\%) & 85 \\
Diabetes mellitus (\%) & 20 \\
\hline Atrial fibrillation (\%) & 36 \\
Coronary artery disease (\%) & 26 \\
\hline Current smoking (\%) & 22 \\
\hline
\end{tabular}

*Significant change from admission to discharge $(\mathrm{P}<0.0001$, Wilcoxon rank test). NIHSS, Stroke Scale of the National Institute of Health; mRS, modified Rankin Scale; BI, Barthel index.

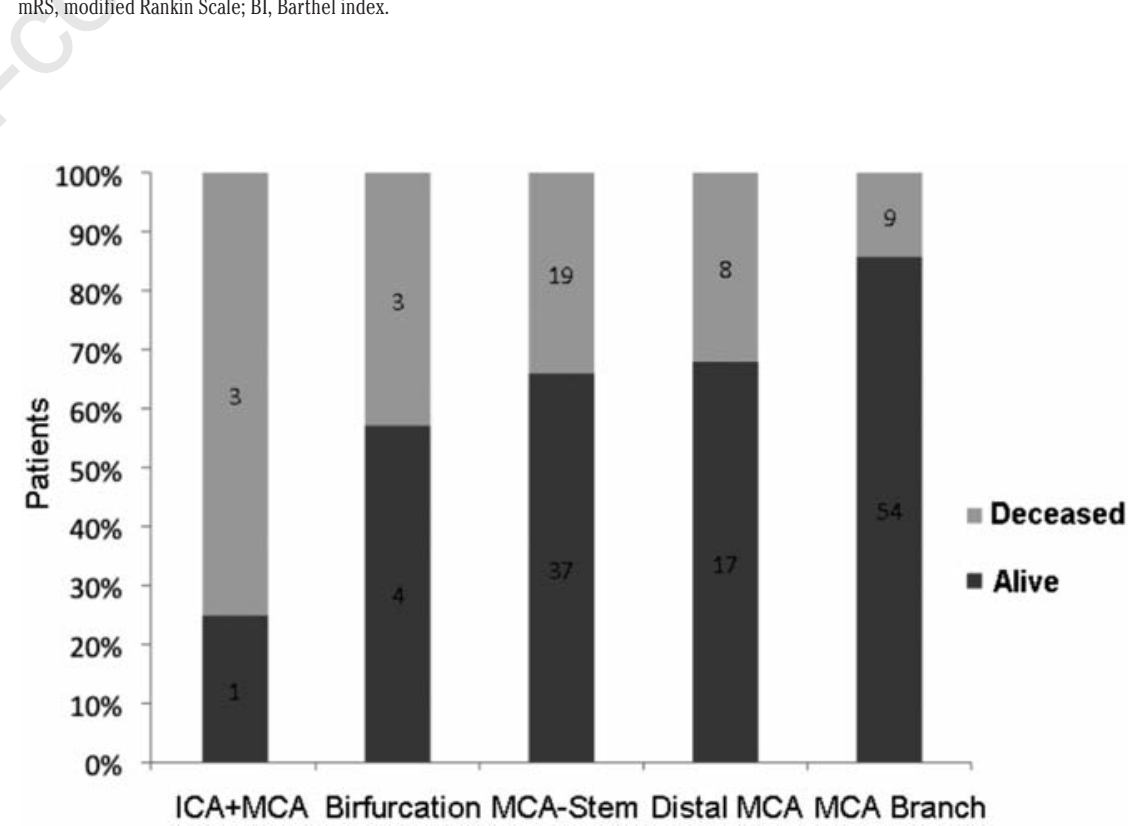

Figure 1. Influence of the location of the occlusion of the middle cerebral artery on the rate of survival. The numbers in each column indicate the number of patients. 
who remained severely impaired or deceased. This was the case both at discharge from the Stroke Unit and for follow-up up to 100 days after stroke. These data accord well with recent reports about intravenous thrombolysis with $0.9 \mathrm{mg}$ rtPA per kilogram body weight in the Safe Implementation of Thrombolysis in Stroke Monitoring Study,22 the Virtual International Stroke Trial Archive, ${ }^{23}$ and the Diffusion and Perfusion Imaging Evaluation for Understanding Stroke Evolution. ${ }^{24}$ These and our data accord with the notion that age and initial NIHSS impact on clinical outcome independent of treatment.

Our rational for the combined use of lowdose rtPA and a subsequent infusion of tirofiban was our attempt to minimize the risk of cerebral hemorrhage on one hand and to counteract arterial reocclusion after stroke and thrombolysis. ${ }^{16}$ Thrombus formation involves the aggregation of activated platelets and the bridging between platelets by fibrinogen via the reconfigured GPIIb/IIIa platelet receptor. ${ }^{25,26}$ The GPIIb/IIIa receptor is a prototypic integrin which is exclusively expressed on the membranes of platelets and megakaryocytes. In experimental stroke models, GPIIb/IIIa antagonists given as only drug or in combination with rtPA have been shown to be safe and effective, since the treated animals had reduced infarct volumes. ${ }^{27,28}$ Clinical studies suggest that tirofiban can disintegrate acute thrombi in the large internal carotid artery and suppress microemboli as detected with transcranial Doppler sonography. ${ }^{29}$ Moreover, in small prospective series we were able to show that a low-dose bolus of $20 \mathrm{mg}$ rtPA and body-weight adjusted infusion of tirofiban resulted in a profound clinical recovery along with recanalization and a decrease in infarct volume.12,17 Similarly, the monoclonocal antibody abciximab that also targets the platelet GPIIb/IIIa receptors was shown to be effective when combined with rtPA in the interventional treatment of vertebrobasilar stroke. ${ }^{30}$ In contrast, abciximab was found to result in an exaggeration of fatal hemorrhages and not to improve outcome in stroke of the anterior circulation. ${ }^{31}$ Since the non-peptide tirofiban was found to be safe in stroke, ${ }^{18}$ it is possible that the monoclonal antibody abciximab blocked the GPIIb/IIIa receptors of all circulating platelets irreversibly precipitating hemorrhagic complications.

In our patients atrial fibrillation was more frequent in the more severely affected patients with more proximal MCA occlusions than in the less severely affected patients. These data highlight the impact of cardioembolic stroke for disability and dependence particularly in the elderly. In contrast, the vascular risk factors were not predictive for poor outcome or discriminating between good recovery and poor recovery or death. It is possible that larg- er cardioembolic thrombi would occlude larger more proximal arteries including the distal ICA and that the thrombi may be more resistant to thrombolysis in older than in younger patients. Both factors would prevent rapid recanalization, early reperfusion and neurological recovery. ${ }^{1-4}$ Since rtPA is beneficial also in the elderly, ${ }^{32,33}$ it should not be withheld from them in acute stroke. But oral anticoagulation because of absolute arrhythmia related to atrial fibrillation is of even greater importance.
In accordance with other recent studies we found that the rate of survival was better the more distal the occlusion of the MCA was. ${ }^{34-37}$ Similar results were obtained concerning the neurological disability and recovery. 17,38 Most likely, this was due to smaller infarcts in more distal cerebral artery occlusions that have been found to exhibit a better recanalization rate. ${ }^{34-39}$ It is tempting to speculate that mechanical devices may be employed in patients with proximal MCA occlusions. The benefit of tirofiban

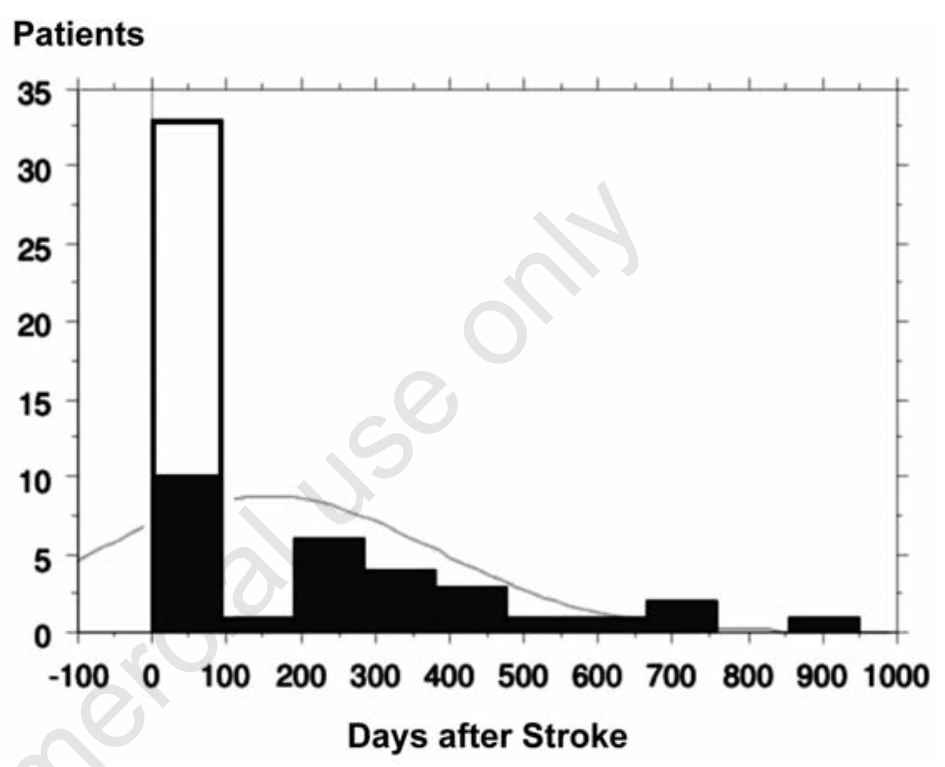

Figure 2. Distribution of dead patients following acute stroke. The majority of patients expired within the first 100 days. Note that 23 patients already died on the Stroke Unit (open column).

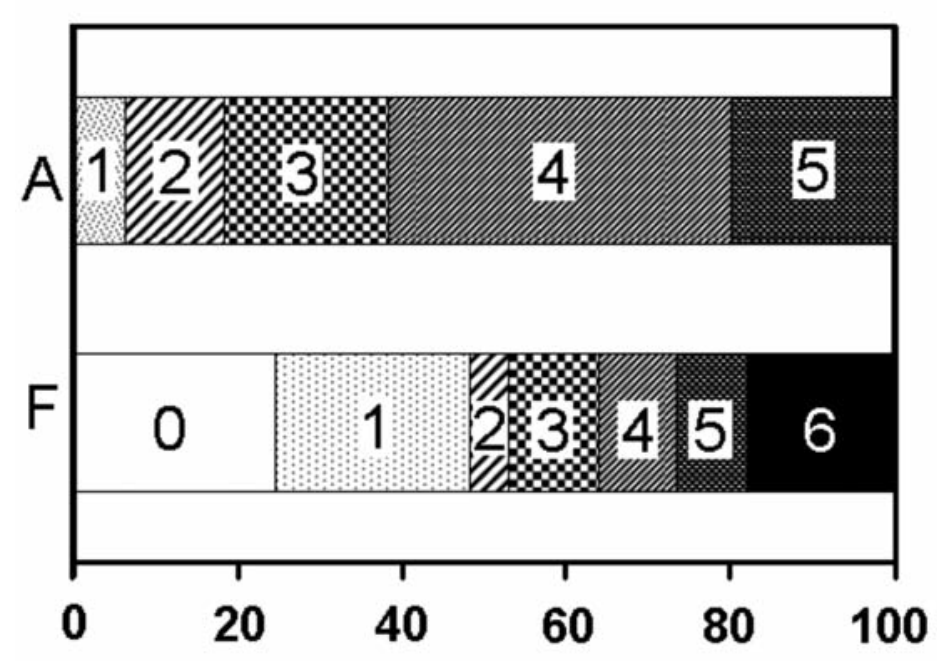

Figure 3. Neurological impairment of the 192 patients as assessed with the modified Rankin Scale on admission before thrombolysis (A) and at follow up (F). Note that half of the patients had no only or little impairment at follow up. ${ }^{20}$ 
in such a situation is that it can be combined with local thrombolysis enhancing its effect beyond the narrow time window of thrombolysis. Thus, it may be of interest in the context of interventional approaches for the treatment of acute stroke.

The rate of fatal hemorrhage in our study was in the range of thrombolysis with $0.9 \mathrm{mg}$ rtPA per kilogram body weight. ${ }^{34-42}$ This accords with our recent observation that tirofiban does not increase the bleeding rate in acute stroke. ${ }^{18}$ It should be noted that in that former study tirofiban was administered as a single drug or in addition to aspirin, while in this study tirofiban was combined with rtPA even in patients receiving clopidogrel hydrogensulfate prior to their present stroke.

\section{Conclusions}

Here, we have shown that the combined use of an intravenous bolus of $20 \mathrm{mg} \mathrm{rtPA}$ and a subsequent body-weight adjusted infusion of tirofiban had an efficacy and safety similarly to systemic thrombolysis with body-weight adjusted rtPA. Our study, however, has limitations. First, we did not perform a direct comparison with the standard thrombolytic treatment with body-weight adjusted rtPA. As evident from this study and calculated from the known effect size, a double-blind, randomized, multi-center trial would require at least 5000 patients per treatment arm. Also, since rtPA has been approved as standard care for acute stroke, a placebo control group was ethically not acceptable. Second, we did not asses the rate of early recanalization in these patients. This will be the topic of a subsequent study. Third, since it is difficult to follow-up patients for face-to-face interviews, we chose to perform the follow-up study using telephone interviews. It has been found previously that both the $\mathrm{BI}$ and $\mathrm{mRS}$ can be used reliably in this way. ${ }^{43,44}$ Fourth, we did not monitor depression or dementia that both have been reported to affect post-stroke recovery and death. 45

\section{References}

1. Hacke W, Kaste M, Bluhmki E, et al. Thrombolysis with alteplase 3 to 4.5 hours after acute ischemic stroke. N Engl J Med 2008;359:1317-29.

2. Delgado-Mederos R, Rovira A, AlvarezSabín J, et al. Speed of tPA-induced clot lysis predicts DWI lesion evolution in acute stroke. Stroke 2007;38:955-60.

3. Zangerle A, Kiechl S, Spiegel M, et al. Recanalization after thrombolysis in stroke patientsp. Predictors and prognos- tic implications. Neurology 2007;68:39-44.

4. Davis S, Donnan G, Parsons M, et al. Effects of alteplase beyond $3 \mathrm{~h}$ after stroke in the Echoplanar Imaging Thrombolytic Evaluation Trial (EPITHET) a placebo-controlled randomised trial. Lancet Neurol 2008;7:299-309.

5. Seitz RJ, Meisel S, Weller P, et al. The initial ischemic event: PWI and ADC for stroke evolution. Radiology 2005;237:1020-8.

6. Bang OY, Saver JL, Buck BH, et al. Impact of collateral flow on tissue fate in acute ischaemic stroke. J Neurol Neurosurg Psychiatry 2008;79:625-9.

7. Kidwell CS, Saver JL, Mattiello J, et al. Thrombolytic reversal of acute human cerebral ischemic injury shown by diffusion/perfusion magnetic resonance imaging. Ann Neurol 2000;47:462-9.

8. Rother J, Schellinger PD, Gass A, et al. Effect of intravenous thrombolysis on MRI parameters and functional outcome in acute stroke $<6$ hours. Stroke 2002;33: 2438-45.

9. Albers GW, Thijs VN, Wechsler L, et al. Magnetic resonance imaging profiles predict clinical response to early reperfusion. The diffusion and perfusion imaging evaluation for understanding stroke evolution (DEFUSE) study. Ann Neurol 2006;60:50817.

10. Lees KR, Bluhmki E, von Kummer R, et al. Time to treatment with intravenous alteplase and outcome in stroke: an updated pooled analysis of ECASS, ATLANTIS, NINDS, and EPITHET trials. Lancet 2010; 375:1695-703.

11. The platelet receptor inhibition in ischemic syndrome management in patients limited by unstable signs and symptoms (PRISM-PLUS) study investigators. Inhibition of the platelet glycoprotein IIb/IIIa receptor with tirofiban in unstable angina and non-Q-wave myocardial infarction. N Engl J Med 1998;338:1488-97

12. Seitz RJ, Siebler M. Platelet GPIIb/IIIa receptor antagonists in human ischemic brain disease. Curr Vasc Pharmacol 2008; 6:29-36.

13. Hacke W, Kaste M, Fieschi C, et al. Randomised double-blind placebo-controlled trial of thrombolytic therapy with intravenous alteplase in acute ischaemic stroke (ECASS II). Second EuropeanAustralasian Acute Stroke Study Investigators. Lancet 1998;352:1245-51.

14. Choudhri TF, Hoh BL, Zerwes HG, et al. Reduced microvascular thrombosis and improved outcome in acute murine stroke by inhibiting GP IIb/IIIa receptor-mediated platelet aggregation. J Clin Invest 1998: 102;1301-10.

15. Abumiya T, Fitridge R, Mazur C, et al. Integrin alpha(IIb) beta(3) inhibitor pre- serves microvascular patency in experimental acute focal cerebral ischemia. Stroke 2000;31:1402-9.

16. Saqquar M, Molina CA, Salem A, et al. Clinical deterioration after intravenous recombinant tissue plasminogen activator treatment: a multicenter transcranial Doppler study. Stroke 2007;38:69-74.

17. Seitz RJ, Hamzavi M, Junghans U, et al. Thrombolysis with recombinant tissue plasminogen activator and tirofiban in stroke. Preliminary observations. Stroke 2003;34:1932-5.

18. Siebler M, Hennerici MG, Schneider D, et al. Safety of tirofiban in acute ischemic stroke. The SaTIS trial. Stroke 2011;42: 2388-92.

19. Brott T, Adams HP Jr, Olinger CP, et al. Measurements of acute cerebral infarctionpp.a clinical examination scale. Stroke 1989;20:864-70.

20. van Swieten JC, Koudstaal PJ, Visser MC, et al. Interobserver agreement for the assessment of handicap in stroke patients. Stroke 1998;19:604-7.

21. Mahoney F, Barthel D. Functional evaluation. The Barthel index. Md State Med J 1965;14:61-5.

22. Wahlgren N, Ahmed N, Eriksson N, et al. Multrivariable analysis of outcome predictors and adjustment of main outcome results to baseline data profile in randomized controlled trials: safe implementation of thrombolysis in stroke-monitoring study (SITS-MOST). Stroke 2008;39:3316-22.

23. Hallevi H, Albright KC, Martin-Schild SB, et al. Recovery after ischemic stroke. Criteria for good outcome by level of disability at day 7 . Cerebrovasc Dis 2009;28: 341-8.

24. Vora NA, Shook SJ, Schumacher HC, et al. A 5-item scale to predict stroke outcome after cortical middle cerebral artery territory infarction: validation from results of the diffusion and perfusion imaging evaluation for understanding stroke evolution (DEFUSE) study. Stroke 2011;42:645-9.

25. Kulkarni S, Dopheide SM, Yap CL, et al. A revised model of platelet aggregation. J Clin Invest 2000;105:783-91.

26. Schwarz M, Meade G, Stoll P, et al. Conformation-specific blockade of the integrin GPIIb/IIIa: a novel antiplatelet strategy that selectively targets activated platelets. Circ Res 2006;99:25-33.

27. Shuaib A, Yang Y, Nakada MT, et al. Glycoprotein IIb/IIIa antagonist, murine 7E3 F(ab')2, and tissue plasminogen activator in focal ischemiapp.evaluation of efficacy and risk of hemorrhage with combination therapy. J Cereb Blood Flow Metab 2002;22:215-22.

28. Meada M, Moriguchi A, Mihara K, et al. FK419, a nonpeptide platelet glycoprotein 
IIb/IIIa antagonist, ameliorates brain infarction associated with thrombotic focal cerebral ischemia in monkeys: comparison with tissue plasminogen activator. J Cereb Blood Flow Metab 2005;25:108-18.

29. Junghans U, Siebler M. Cerebral microembolism is blocked by tirofiban, a selective nonpeptide platelet glycoprotein IIb/IIIa receptor antagonist. Circulation 2003;107: 2717-21.

30. Eckert B, Koch C, Thomalla G, et al. Aggressive therapy with intravenous abciximab and intra-arterial rtPA and additional PTA/stenting improves clinical outcome in acute vertebrobasilar occlusion: combined local fibrinolysis and intravenous abciximab in acute vertebrobasilar stroke treatment (FAST): results of a multicenter study. Stroke 2005;36:1160-5.

31. Adams HP Jr, Effron MB, Torner J, et al. Emergency administration of abciximab for treatment of patients with acute ischemic stroke: results of an international phase III trialpp.abciximab in emergency treatment of stroke trial (AbESTTII). Stroke 2008;39:87-99.

32. Mishra NK, Ahmed N, Andersen G, et al. Thrombolysis in very elderly people: controlled comparison of SITS international stroke thrombolysis registry and virtual international stroke trials archive. Br Med J 2010;341:c6046.
33. Ford GA, Ahmed N, Azevedo E, et al. Intravenous alteplase for stroke in those older than 80 years old. Stroke 2010;41: 2568-74.

34. Kim YS, Garami Z, Mikulik R, et al. Early recanalization rates and clinical outcomes in patients with tandem internal carotid/middle cerebral artery occlusion and isololated middle cerebral artery occlusion. Stroke 2005;36:869-71.

35. Wunderlich MT, Goertler M, Postert T, et al. Recanalization after intravenous thrombolysis: does a recanalization time window exist? Neurology 2007;68:1364-8.

36. Fiehler J, Knudson K, Thomalla G, et al. Vascular occlusion sites determine differences in lesion growth from early apparent diffusion coefficient lesion to final infarct. Am J Neuroradiol 2005;26:1056-61.

37. Derex L, Hermier M, Adeleine P, et al. Influence of the site of arterial occlusion on multiple baseline hemodynamic MRI parameters and post-thrombolytic recanalization in acute stroke. Neuroradiology 2004;46:883-7.

38. Hacke W, Donnan G, Fieschi C, et al. Association of outcome with early stroke treatmentpp.pooled analysis of ATLANTIS, ECASS, and NINDS rt-PA stroke trials. Lancet 2004;363:768-74.

39. Seitz RJ, Oberstrass H, Ringelstein A, et al. Failed recovery from thrombolysis is pre- dicted by the initial DWI lesion. Cerebrovasc Dis 2011;31:580-7.

40. Blinzler C, Breuer L, Huttner HB, et al. Characteristics and outcome of patients with early complete neurological recovery after thrombolysis for acute ischemic stroke. Cerebrovasc Dis 2010;31:185-90.

41. Fonarow G, Reeves M, Zhao X, et al. Get wth the guidelines-stroke steering committee and investigators. Age-related differences in characteristics, performance measures, treatment trends, and outcomes in patients with ischemic stroke. Circulation 2010;121:879-91.

42. Arnold M, Halpern M, Meier N, et al. Agedependent differences in demographics, risk factors, co-morbidity, etiology, management, and clinical outcome of acute ischemic stroke. J Neurol 2008;255:1503-7.

43. Korner-Bitensky N, Wodd-Dauphinee S. Barthel index information elicited over the telepone. Is ist reliable? Amer J Phys Med Rehabil 1995;74:9-18.

44. Janssen PM, Visser NA, Dorhout Mees SM, et al. Comparison of telephone and face-toface assessment of the modified Rankin Scale. Cerebrovasc Dis 2010;29:137-9.

45. Pendlebury S, Rothwell P. Prevalence, incidence, and factors associated with prestroke and post-stroke dementiapp.a systematic review and meta-analysis. Lancet Neurol 2009;8:1006-18. 